\title{
Avaliação do resultado de um programa educativo dirigido a paraplégicos visando o autocuidado relacionado aos déficits identificados na eliminação intestinal *
}

\author{
Effect of an educational program in intestinal elimination self-care deficits among individuals with \\ paraplegia
}

Evaluación del resultado de un programa educativo dirigido a parapléjicos visando el auto cuidado relacionado a los déficits identificados en la eliminación intestinal

\section{Marcele Pescuma Capeletti Padula ${ }^{1}$, Mariana Fernandes de Souza $^{2}$}

\section{RESUMO}

Objetivos: Avaliar o progresso de paraplégicos na assunção do autocuidado, após a implementação de um programa educativo relacionado a 16 déficits identificados na eliminação intestinal; verificar a influência do nível neurológico da lesão e lócus de controle à saúde na assunção do autocuidado. Métodos: Estudo semi-experimental com 28 pacientes, 14 pertencentes ao grupo experimental aos quais aplicou-se o programa educativo e 14 do grupo controle submetidos ao tratamento convencional da instituição. Foram atribuídos escores às ações de enfermagem: fazer -4 , guiar -3 , apoiar -2 , ensinar -1 , independente -0 . Resultados: O grupo experimental progrediu significativamente na assunção do autocuidado em comparação com o grupo controle (teste de Quiquadrado p-value<0,05). O nível neurológico da lesão e o lócus de controle à saúde não influenciaram os resultados (testes Mann-Whitney e Quiquadrado). Conclusão: Os paraplégicos submetidos ao programa educativo conseguiram progredir na assunção do autocuidado.

Descritores: Educação em saúde; Paraplegia; Educação do paciente; Autocuidado; Defecação; Cuidados de enfermagem

\begin{abstract}
Objectives: To evaluate self-care progress of individuals with paraplegia who attended an educational program regarding 16 intestinal elimination self-care deficits, and to determine the influence of the neurological level of the injury and locus of control on the progress of the self-care. Methods: A quasi-experimental design was used to conduct the study. The sample consisted of 28 individuals with paraplegia who were assign to usual care group $(\mathrm{N}=14)$ or to the educational program in intestinal elimination self-care deficits group (N-14). Nursing interventions were scored "Do $=4$ ", "Guide $=3$ ", "Support $=2$ ", "Teach $=1$ ", "Independent $=0$ ". Results: Individuals with paraplegia who attended the educational program had better progress toward self-care than those to the usual care group $(\mathrm{p}<0.05)$. The neurological level of the injury and the locus of control did not influence individuals' progress toward self-care. Conclusion: The individuals who attended the educational program progressed satisfactorily toward intestinal elimination self-care actions.
\end{abstract}

Keywords: Health education; Paraplegia; Patient education; Self care; Defecation; Nursing care

\section{RESUMEN}

Objetivos: Evaluar el progreso de los pacientes parapléjicos en la asunción del auto cuidado después de la implantación de un programa educativo relacionado a 16 déficits identificados en la eliminación intestinal; verificar la influencia del nivel neurológico de lesión y locus de control a la salud en la asunción del auto cuidado. Métodos: Estudio cuasi experimental realizado con 28 pacientes, 14 pertenecientes al grupo experimental a quienes se les aplicó el programa educativo y 14 del grupo control sometidos al tratamiento convencional de la institución. Fueron atribuidos puntuaciones a las acciones de enfermería: hacer -4 , guiar -3 , apoyar -2 , enseñar -1 , independiente -0 . Resultados: El grupo experimental progresó significativamente en la asunción del auto cuidado en comparación al grupo control (test de Chi cuadrado p-value $<0,05$ ). El nivel neurológico de la lesión y el lócus de control a la salud no influyeron en los resultados (tests Mann-Whitney y Chi cuadrado). Conclusión: Los parapléjicos sometidos al programa educativo consiguieron progresar en la asunción del auto cuidado. Descriptores: Educación en salud; Paraplejia; Educación del paciente; Autocuidado; Defecación; Cuidados de enfermería

* Artigo extraído da tese de doutorado "Avaliação do resultado de um programa educativo dirigido a paraplégicos visando o autocuidado relacionado aos déficits identificados na eliminação intestinal”, apresentada ao programa de Pós-Graduação em Enfermagem da Universidade Federal de São Paulo - UNIFESP - São Paulo (SP), Brasil.

${ }^{1}$ Professora Doutora; Docente do Curso de Graduação em Enfermagem da Faculdade de Ciências Médicas da Santa Casa de São Paulo. São Paulo (SP), Brasil. ${ }^{2}$ Professora Titular do Departamento de Enfermagem da Universidade Federal de São Paulo - UNIFESP-São Paulo (SP), Brasil 


\section{INTRODUÇÃO}

A lesão na medula espinhal é um agravo que resulta em sérias alterações das funções motoras, sensitivas e dos sistemas orgânicos, uma vez que é a principal via condutora de informações entre o corpo e o cérebro. Dada sua gravidade, causa grandes modificações na vida do paciente, da família e da sociedade. A lesão traumática da medula espinhal resultante de uma agressão física inesperada é a mais freqüente.

Nos Estados Unidos da América, o trauma raquimedular é um importante problema de saúde afetando 200.000 a 500.000 pessoas, com uma estimativa de 10.000 novas lesões acontecendo a cada ano ${ }^{(1)}$.

As [...]"lesões acima do segmento medular T1 causam tetra ou quadriplegia, reservando-se o nome paraplegia para aquelas que comprometem os grandes segmentos medulares localizados abaixo de T1. O nível de lesão é determinado pelo último segmento sensitivo e/ou motor preservado"(2).

Além do dano sensitivo e motor, a lesão medular leva, também, a alterações nas eliminações urinária e fecal devidas à perda dos controles esfincterianos vesical e anal com conseqüente mudança no padrão dessas eliminações. A impossibilidade do controle esfincteriano vesical traz vários problemas para o paciente, entre eles, a não aceitação social, a limitação do programa de reabilitação e complicações clínicas como as infecções urinárias, litíases vesicais e hidronefrose.

Em relação à eliminação intestinal, o lesado medular poderá apresentar dois problemas principais: “[...]a incontinência fecal, ou a dificuldade de conter as fezes dentro do reto, por falha esfincteriana, e a constipação, ou a demora da eliminação fecal, causando desconforto além de outros distúrbios neurogênicos" (3).

Em nossa experiência profissional, constatamos que os problemas dos lesados medulares relativos à eliminação intestinal são extremamente constrangedores e limitantes, uma vez que quando ocorre um episódio de incontinência fecal no centro de reabilitação, o paciente precisa parar a atividade que estava sendo desenvolvida naquele momento para realizar a sua higiene pessoal e troca de roupas sujas. Caso o paciente apresente obstipação, além da incômoda distensão abdominal, ele poderá apresentar a qualquer momento, sinais de crise autonômica hipertensiva, evento grave e comprometedor que necessita de atendimento de urgência e que poderá levá-lo a outras seqüelas e até a morte, se ignorado.

Assim, visando a reintegração do lesado medular à comunidade e utilizando conhecimentos científicos da enfermagem para esse fim, selecionamos, para este trabalho, indivíduos paraplégicos, que constituem a maioria dos lesados medulares, a seqüela de disfunção intestinal e os déficits de autocuidado correspondentes, como aspectos que acreditamos necessários e possíveis de serem trabalhados para o autocuidado do indivíduo, e assim contribuir para a sua reabilitação. Para guiar a elaboração deste trabalho, escolhemos o modelo conceitual de autocuidado de Dorothea E. Orem ${ }^{(4)}$.

Perante os déficits de autocuidado relacionados à eliminação intestinal em pacientes paraplégicos, passamos a questionar: Será que um conjunto de procedimentos elaborados especificamente para auxiliá-los na assunção do autocuidado, poderia ajudá-los neste sentido? A esta questão procuramos responder neste estudo.

\section{OBJETIVOS}

\section{Objetivo Geral}

Avaliar o resultado de um Programa educativo dirigido a pacientes paraplégicos visando o autocuidado relacionado aos déficits na eliminação intestinal.

\section{Objetivos específicos}

Caracterizar a amostra segundo as variáveis: nível neurológico da lesão, locus de controle à saúde; avaliar o progresso dos pacientes paraplégicos na assunção do autocuidado após a implementação de um programa educativo relacionado aos 16 déficits identificados na eliminação intestinal; verificar a influência das variáveis antecedentes: nível neurológico da lesão e locus de controle à saúde na assunção do autocuidado.

\section{REFERENCIAL TEÓRICO}

O modelo conceitual do autocuidado elaborado por Dorothea E. Orem ${ }^{(4)}$ tem como idéia central que as pessoas em sua vida diária aprendem e executam ações reguladoras para si próprias e para outros que delas dependam, a fim de manter a vida, a saúde e o bemestar. Isto é, as pessoas mantêm seu autocuidado e o provêm para seus dependentes. Quando as demandas de autocuidado ultrapassam a capacidade do indivíduo para atendê-las surgem os déficits de autocuidado.

Em situações de déficit de autocuidado por incapacidade de cuidar de si, ou de seus dependentes, em estados de necessidades relacionadas à saúde, a enfermagem surge como um serviço humano, profissional com capacidade para agenciar, prover as demandas de autocuidado terapêutico, regular e ajudar os indivíduos na capacidade de agenciar o próprio cuidado.

A enfermagem, na implementação de seu plano de cuidados, pode seguir cinco métodos de ajuda: 1 - fazer pelo outro, 2- guiar e dirigir, 3- prover apoio físico e psicológico, 4- prover e manter um ambiente que favoreça o desenvolvimento e 5- ensinar e educar.

Para este estudo estabelecemos as seguintes ações: 
Fazer- uso de habilidades próprias para conseguir resultados específicos para a pessoa incapaz de conseguílos por si mesma (escore 4); Guiar- dirigir ou supervisionar nas situações em que a pessoa deve fazer escolhas, deliberar entre um e outro procedimento ensinado (escore 3); Apoiar- executar ações de prevenção para que a pessoa não falhe nas escolhas ou decisões tomadas, assim como o apoio físico e emocional, ajudando nas reflexões das situações (escore 2); Ensinar- instruir as pessoas que necessitam desenvolver conhecimento ou habilidades (escore 1); Independente- paciente não necessita de qualquer método de ajuda, foi categorizado como independente (escore zero).

Os déficits de autocuidado relacionados à Eliminação Intestinal selecionados para este estudo foram: Déficits relacionados à ingestão alimentar: 1- freqüência alimentar alterada, 2- qualidade inadequada dos alimentos ingeridos, 3- ingestão inadequada de líquidos. Déficits relacionados ao funcionamento intestinal: 4- ausência ou insuficiência de conhecimento do padrão normal de eliminação intestinal, 5- mudança no padrão de eliminação intestinal: perda involuntária de fezes/constipação, aumento/ diminuição na freqüência de eliminação intestinal, 6mudança do local utilizado para evacuação/ posicionamento inadequado para evacuação, 7- uso de laxantes não-naturais e/ou enemas, 8- alteração do aspecto das fezes eliminadas. Déficits relacionados à capacidade de movimentação: 9- higiene íntima inadequada após eliminação intestinal/ necessidade do auxílio de outra pessoa para higiene íntima após eliminações intestinais, 10 - necessidade do auxílio de outra pessoa para posicionamento adequado para a evacuação, transferências e transporte até o local de evacuação, 11necessidade do auxílio de outra pessoa para a limpeza da comadre, lavagem de fraldas de pano e troca de fraldas, 12- uso inadequado de fraldas, 13- uso da comadre de forma inadequada, 14- lavagem inadequada de fraldas de pano, 15- limpeza inadequada da comadre. Ausência ou insuficiência de conhecimento sobre a crise autonômica hipertensiva: 16- desconhece as causas mais freqüentes, desconhece os sinais e sintomas e desconhece as condutas adequadas na vigência da crise.

\section{HIPÓTESE}

Paraplégicos submetidos a um Programa educativo visando o autocuidado relacionado aos déficits identificados na eliminação intestinal conseguem progredir na assunção do autocuidado.

\section{Variável independente}

Programa educativo dirigido a paraplégicos visando o autocuidado relacionado aos déficits identificados na eliminação intestinal. Este programa visou preparar indivíduos paraplégicos para o autocuidado relacionado à eliminação intestinal. Foi elaborado fundamentado nos déficits de autocuidado nesta área, identificado em pacientes paraplégicos, tendo como base bibliografia específica, a Teoria de Enfermagem de Orem, assim como nossa experiência na assistência de enfermagem a estes pacientes.

\section{Variável dependente}

Progresso dos paraplégicos na assunção do autocuidado relacionado à eliminação intestinal. Operacionalmente, foi verificado: a) por escores atribuídos às ações de enfermagem, segundo os métodos de ajuda que o paciente requereu em cada déficit de autocuidado identificado e b) em cada déficit, pelo progresso dos paraplégicos na assunção do autocuidado relacionado à eliminação intestinal, segundo as categorias: progrediu, não alterou, regrediu.

\section{Variáveis antecedentes}

Nível neurológico da lesão e o locus de controle à saúde.

\section{MÉTODOS}

Trata-se de um delineamento semi-experimental em que os sujeitos da pesquisa foram alocados aleatoriamente em dois grupos: controle e experimental.

O estudo foi realizado em dois centros de reabilitação da cidade de São Paulo, o Lar Escola São Francisco e a Irmandade Santa Casa de Misericórdia de São Paulo.

\section{População e amostra}

A população constituiu-se de indivíduos paraplégicos, de ambos os sexos, com idades entre 18 e 60 anos, que estavam iniciando o tratamento de reabilitação nos locais de estudo.

\section{Coleta de dados}

Foram utilizados para a coleta de dados os seguintes instrumentos:

Medida do Locus de Controle à Saúde: utilizamos a Escala Multidimensional de Locus de Controle à Saúde que foi adaptada para língua portuguesa por Marília Dela Coleta em 1995. Optamos pelo uso da escala autoadministrável, com indivíduos alfabetizados.

Avaliação dos Déficits de Autocuidado relacionados à Eliminação Intestinal: na parte 1 consta a data da avaliação, iniciais do nome do paciente e os dados sobre as variáveis antecedentes; na parte 2 constam dados sobre a identificação dos déficits.

Parâmetros para avaliação dos déficits e das ações de enfermagem: foram considerados padrões para emissão de juízos avaliativos e construídos com base no 
Referencial Teórico e na caracterização dos déficits.

Identificação dos déficits relacionados à eliminação intestinal e dos métodos de ajuda de enfermagem: contém os 16 déficits a serem identificados em cada paciente e os métodos de ajuda de enfermagem correspondentes, com o respectivo escore.

\section{Procedimentos}

O projeto foi enviado ao Comitê de Ética em Pesquisa da Universidade Federal de São Paulo - UNIFESP e ao Comitê de Ética e Pesquisa em Seres Humanos da Irmandade Santa Casa de Misericórdia de São Paulo tendo sido aprovado em ambos.

Os pacientes da amostra foram sorteados para alocação nos grupos experimental ou controle. A seguir, fizemos os esclarecimentos sobre a aceitação livre e garantia de anonimato; os que aceitaram, assinaram o Termo de Consentimento Livre e Esclarecido.

A aplicação dos instrumentos nos grupos foi feita de forma individualizada. Primeiramente foi aplicado o Instrumento de Medida do Locus de Controle à Saúde. Após, demos continuidade à avaliação inicial com a consulta de enfermagem que teve a duração aproximada de 60 minutos quando, então, preenchíamos o Instrumento de Avaliação dos Déficits de Autocuidado relacionados à Eliminação Intestinal.

Com base nos Parâmetros para avaliação dos déficits e das ações de enfermagem identificamos em cada déficit apresentado pelos pacientes, as ações de enfermagem ou métodos de ajuda correspondentes, registrando-as no Instrumento para Identificação dos Déficits relacionados à Eliminação Intestinal e dos Métodos de Ajuda de Enfermagem.

$\mathrm{Na}$ semana seguinte, iniciamos a aplicação do Programa educativo dirigido a paraplégicos, visando o autocuidado relacionado aos déficits identificados na eliminação intestinal, a cada paciente do grupo experimental.

Acompanhamos cada um dos pacientes dos grupos por um período de oito semanas. $\mathrm{Na}$ oitava consulta foi feita a avaliação final dos grupos. Identificamos em cada déficit apresentado pelo paciente os métodos de ajuda de enfermagem correspondentes.

\section{RESULTADOS}

\section{Caracterização dos pacientes da amostra}

A amostra constituiu-se de 28 pacientes, sendo 14 pertencentes ao grupo experimental e 14 pertencentes ao grupo controle. A maioria dos pacientes (11 no total da amostra) apresentou nível neurológico de lesão torácica alta (T1 a T6). No grupo controle, seis pacientes apresentaram lesão torácica alta e no grupo experimental, encontramos seis pacientes com lesão torácica média e baixa
(T7 a T11). Em relação ao locus de controle à saúde, observamos que em treze pacientes da amostra o lócus de controle é interno e, igualmente, em treze pacientes o lócus de controle é externo. O grupo controle apresentou a maioria dos pacientes (8) com locus de controle externo e o grupo experimental apresentou sete pacientes com lócus de controle interno. Neste grupo houve dois pacientes cujos escores foram iguais, tanto para internalidade quanto para externalidade e para análise estatística não foram considerados.

\section{Verificação da homogeneidade dos grupos da} amostra

Utilizamos os testes de Mann-Whitney e Quiquadrado ( $p$-value $<0,05)$ mostrando que houve homogeneidade entre os grupos controle e experimental da amostra estudada.

\section{Resultados relativos ao progresso dos} paraplégicos na assunção do autocuidado

a) Escores atribuídos às ações de enfermagem, segundo os métodos de ajuda que o paciente requeria em cada déficit de autocuidado identificado.

$\mathrm{Na}$ avaliação inicial verificamos quantos pacientes requeriam os métodos de ajuda Ensinar (1), Apoiar (2), Guiar (3), Fazer (4) e quantos estavam Independentes (0) em cada déficit de autocuidado e em cada um dos grupos controle e experimental. Utilizamos o teste Quiquadrado ( $p$-value $<0,05)$ na comparação entre os grupos, e verificamos que os grupos estavam em situação semelhante de dependência (Tabela 1).

Em relação ao déficit 5, todos os pacientes do grupo experimental estavam no método de ajuda fazer, enquanto no grupo controle, os pacientes estavam distribuídos entre os demais métodos de ajuda, revelando que o grupo experimental estava, inicialmente, mais dependente neste aspecto. Esclarecemos que, em nossa pesquisa, o déficit 14 não demandou qualquer ação de enfermagem, uma vez que todos os pacientes deste estudo faziam uso de fraldas descartáveis.

Decorrido o tempo de aplicação do programa educativo aos pacientes do grupo experimental, ambos os grupos foram submetidos à avaliação final (Tabela 2).

$\mathrm{Na}$ avaliação final, de acordo com o teste Quiquadrado ( $\mathrm{p}$-value $<0,05)$ foram observadas diferenças estatisticamente significantes em relação aos déficits 1, 2, 3, 4, 5, 8 e 16 mostrando que mais pacientes do grupo experimental encontravam-se nas categorias de menos dependência da enfermagem. Nos outros déficits $6,7,9,11,12,13$ e 15 não houve diferença significativa entre os dois grupos na avaliação final, com os pacientes evoluindo na assunção do autocuidado da mesma forma em ambos.

b) Progresso dos paraplégicos na assunção do autocuidado relacionado à eliminação intestinal, segundo 
as categorias progrediu, não alterou e regrediu.

Tabela 1 - Paraplégicos dos grupos controle e experimental, segundo os métodos de ajuda de enfermagem identificados na avaliação inicial, em cada um dos déficits de autocuidado relacionado à eliminação intestinal. São Paulo, SP, 2002.

\begin{tabular}{|c|c|c|c|c|c|c|}
\hline \multirow{3}{*}{ Déficits } & \multirow{3}{*}{$\begin{array}{l}\text { Métodos } \\
\text { de ajuda }\end{array}$} & \multicolumn{4}{|c|}{ Grupos } & \multirow{3}{*}{$\begin{array}{c}\text { Nível } \\
\text { descritiv } \\
\text { (p-value) }\end{array}$} \\
\hline & & \multicolumn{2}{|c|}{ Controle } & \multicolumn{2}{|c|}{ Experimental } & \\
\hline & & $\mathbf{N}$ & $\%$ & $\mathbf{N}$ & $\overline{\%}$ & \\
\hline \multirow[t]{3}{*}{ D1(inicial) } & Ensinar & 6 & 42,86 & 4 & 28,57 & 0,287 \\
\hline & Apoiar & 3 & 21,43 & 7 & 50,00 & \\
\hline & Guiar & 5 & 35,71 & 3 & 21,43 & \\
\hline \multirow[t]{3}{*}{ D2(inicial) } & Ensinar & 1 & 7,14 & 2 & 14,28 & 0,815 \\
\hline & Apoiar & 7 & 50,00 & 6 & 42,86 & \\
\hline & Guiar & 6 & 42,86 & 6 & 42,86 & \\
\hline \multirow[t]{3}{*}{ D3 (inicial) } & Ensinar & 3 & 21,43 & 1 & 7,14 & 0,535 \\
\hline & Apoiar & 9 & 64,29 & 10 & 71,43 & \\
\hline & Guiar & 2 & 14,28 & 3 & 21,43 & \\
\hline \multirow[t]{2}{*}{ D4(inicial) } & Ensinar & 10 & 71,43 & 9 & 64,29 & 0,500 \\
\hline & Apoiar & 4 & 28,57 & 5 & 35,71 & \\
\hline \multirow[t]{4}{*}{$\overline{\text { D5 (inicial) }}$} & Ensinar & 2 & 14,29 & - & - & 0,054 \\
\hline & Apoiar & 2 & 14,29 & - & - & \\
\hline & Guiar & 2 & 14,29 & - & - & \\
\hline & Fazer & 8 & 57,13 & 14 & 100,00 & \\
\hline \multirow[t]{3}{*}{ D6 (inicial) } & Ensinar & 10 & 71,43 & 7 & 50,00 & 0,171 \\
\hline & Apoiar & - & - & 3 & 21,43 & \\
\hline & Guiar & 4 & 28,57 & 4 & 28,57 & \\
\hline \multirow[t]{3}{*}{ D7 (inicial) } & Ensinar & 10 & 71,43 & 8 & 57,14 & 0,329 \\
\hline & Apoiar & - & - & 2 & 14,29 & \\
\hline & Guiar & 4 & 28,57 & 4 & 28,57 & \\
\hline \multirow[t]{2}{*}{ D8 (inicial) } & Ensinar & 3 & 21,43 & 2 & 14,29 & 0,500 \\
\hline & Apoiar & 11 & 78,57 & 12 & 85,71 & \\
\hline \multirow[t]{4}{*}{$\overline{\text { D9 (inicial) }}$} & Ensinar & 8 & 57,14 & 7 & 50,00 & 0,494 \\
\hline & Apoiar & 4 & 28,57 & 2 & 14,29 & \\
\hline & Guiar & 2 & 14,29 & 4 & 28,57 & \\
\hline & Fazer & - & - & 1 & 7,14 & \\
\hline \multirow[t]{4}{*}{ D10 (inicial) } & Ensinar & 4 & 28,57 & 5 & 35,72 & 0,375 \\
\hline & Apoiar & 2 & 14,29 & - & - & \\
\hline & Guiar & 8 & 57,14 & 8 & 57,14 & \\
\hline & Fazer & - & - & 1 & 7,14 & \\
\hline \multirow[t]{4}{*}{ D11(inicial) } & $\begin{array}{l}\text { Independ } \\
\text { ente }\end{array}$ & 4 & 28,57 & 4 & 28,57 & 0,172 \\
\hline & Ensinar & 2 & 14,29 & - & - & \\
\hline & Apoiar & 7 & 50,00 & 5 & 35,71 & \\
\hline & Guiar & 1 & 7,14 & 5 & 35,71 & \\
\hline \multirow[t]{3}{*}{ D12(inicial) } & $\begin{array}{l}\text { Independ } \\
\text { ente }\end{array}$ & 4 & 28,57 & 3 & 21,43 & 0,088 \\
\hline & Ensinar & 9 & 64,29 & 5 & 35,71 & \\
\hline & Apoiar & 1 & 7,14 & 6 & 42,86 & \\
\hline \multirow[t]{3}{*}{ D13(inicial) } & $\begin{array}{l}\text { Independ } \\
\text { ente }\end{array}$ & 12 & 85,71 & 8 & 57,15 & 0,214 \\
\hline & Ensinar & 2 & 14,29 & 5 & 35,71 & \\
\hline & Apoiar & - & - & 1 & 7,14 & \\
\hline D14(inicial) & $\begin{array}{l}\text { Independ } \\
\text { ente }\end{array}$ & 14 & 100,00 & 14 & 100,00 & ** \\
\hline \multirow[t]{3}{*}{ D15(inicial) } & $\begin{array}{l}\text { Independ } \\
\text { ente }\end{array}$ & 12 & 85,72 & 8 & 57,15 & 0,177 \\
\hline & Ensinar & 1 & 7,14 & 5 & 35,71 & \\
\hline & Apoiar & 1 & 7,14 & 1 & 7,14 & \\
\hline D16(inicial) & Guiar & 14 & 100,00 & 14 & 100,00 & $* * *$ \\
\hline
\end{tabular}

Tabela 2 - Paraplégicos dos grupos controle e experimental, segundo os métodos de ajuda de enfermagem identificados na avaliação final, em cada um dos déficits de autocuidado relacionado à eliminação intestinal. São Paulo, SP, 2002.

\begin{tabular}{|c|c|c|c|c|c|c|}
\hline \multirow{3}{*}{ Déficits } & \multirow{3}{*}{ Métodos de ajuda } & \multicolumn{4}{|c|}{ Grupos } & \multirow{3}{*}{$\begin{array}{c}\text { Nível } \\
\text { descritivo } \\
\text { (p-value) }\end{array}$} \\
\hline & & \multicolumn{2}{|c|}{ Controle } & \multicolumn{2}{|c|}{ Experimental } & \\
\hline & & $\mathbf{N}$ & $\%$ & $\mathbf{N}$ & $\%$ & \\
\hline \multirow[t]{3}{*}{ D1 (final) } & Independente & 5 & 35,71 & 10 & 71,43 & 0,056 \\
\hline & Apoiar & 5 & 35,71 & 4 & 28,57 & \\
\hline & Guiar & 4 & 28,58 & - & - & \\
\hline \multirow[t]{4}{*}{ D2 (final) } & Independente & 1 & 7,14 & 6 & 42,86 & 0,057 \\
\hline & Ensinar & 1 & 7,14 & 2 & 14,28 & \\
\hline & Apoiar & 9 & 64,29 & 6 & 42,86 & \\
\hline & Guiar & 3 & 21,43 & - & - & \\
\hline \multirow[t]{3}{*}{ D3 (final) } & Independente & 6 & 42,86 & 12 & 85,71 & 0,043 \\
\hline & Apoiar & 5 & 35,71 & 2 & 14,29 & \\
\hline & Guiar & 3 & 21,43 & - & - & \\
\hline \multirow[t]{3}{*}{ D4 (final) } & Independente & 4 & 28,58 & 11 & 78,57 & 0,012 \\
\hline & Ensinar & 5 & 35,71 & 3 & 21,43 & \\
\hline & Apoiar & 5 & 35,71 & - & - & \\
\hline \multirow[t]{5}{*}{ D5 (final) } & Independente & 4 & 28,57 & 9 & 64,29 & 0,005 \\
\hline & Ensinar & - & - & 4 & 28,57 & \\
\hline & Apoiar & 5 & 35,72 & - & - & \\
\hline & Guiar & 1 & 7,14 & 1 & 7,14 & \\
\hline & Fazer & 4 & 28,57 & - & - & \\
\hline \multirow[t]{3}{*}{ D6 (final) } & Independente & 11 & 78,57 & 13 & 92,86 & 0,125 \\
\hline & Apoiar & - & - & 1 & 7,14 & \\
\hline & Guiar & 3 & 21,43 & - & - & \\
\hline \multirow[t]{3}{*}{ D7 (final) } & Independente & 12 & 85,72 & 14 & 100,00 & 0,341 \\
\hline & Apoiar & 1 & 7,14 & - & - & \\
\hline & Guiar & 1 & 7,14 & - & - & \\
\hline \multirow[t]{3}{*}{$\overline{D 8 \text { (final) }}$} & Independente & 4 & 28,57 & 10 & 71,43 & 0,025 \\
\hline & Ensinar & - & - & 1 & 7,14 & \\
\hline & Apoiar & 10 & 71,43 & 3 & 21,43 & \\
\hline \multirow[t]{3}{*}{ D9 (final) } & Independente & 9 & 64,29 & 9 & 64,29 & 0,574 \\
\hline & Apoiar & 4 & 28,57 & 5 & 35,71 & \\
\hline & Guiar & 1 & 7,14 & - & - & \\
\hline \multirow[t]{3}{*}{$\overline{\mathrm{D} 10 \text { (final) }}$} & Independente & 4 & 28,57 & 7 & 50,00 & 0,470 \\
\hline & Apoiar & 2 & 14,29 & 2 & 14,29 & \\
\hline & Guiar & 8 & 57,14 & 5 & 35,71 & \\
\hline \multirow[t]{4}{*}{ D11 (final) } & Independente & 7 & 50,00 & 7 & 50,00 & 0,392 \\
\hline & Ensinar & - & - & 1 & 7,14 & \\
\hline & Apoiar & 6 & 42,86 & 3 & 21,43 & \\
\hline & Guiar & 1 & 7,14 & 3 & 21,43 & \\
\hline \multirow[t]{3}{*}{ D12 (fianl) } & Independente & 6 & 42,86 & 5 & 35,71 & 0,809 \\
\hline & Ensinar & 7 & 50,00 & 7 & 50,00 & \\
\hline & Apoiar & 1 & 7,14 & 2 & 14,29 & \\
\hline \multirow[t]{2}{*}{ D13 (final) } & Independente & 12 & 85,71 & 10 & 71,43 & 0,324 \\
\hline & Ensinar & 2 & 14,29 & 4 & 28,57 & \\
\hline D14 (final) & Independente & 14 & 100,00 & 14 & 100,00 & $* * *$ \\
\hline \multirow[t]{2}{*}{$\overline{\mathrm{D} 15 \text { (final) }}$} & Independente & 13 & 92,86 & 14 & 100,00 & 0,500 \\
\hline & Apoiar & 1 & 7,14 & - & - & \\
\hline D16 (final) & Independente & - & - & 3 & 21,43 & 0,003 \\
\hline & Ensinar & - & - & 6 & 42,86 & \\
\hline & Apoiar & 1 & 7,14 & 1 & 7,14 & \\
\hline & Guiar & 13 & 92,86 & 4 & 28,57 & \\
\hline
\end{tabular}


Apurou-se a evolução dos pacientes dos grupos controle e experimental verificando se o paciente progrediu dentro dos métodos de ajuda. Utilizamos o teste Quiquadrado (p-value<0,05) (Tabela 3).

Tabela 3 - Paraplégicos dos grupos controle e experimental segundo a evolução nos déficits de autocuidado relacionados à eliminação intestinal na avaliação final. São Paulo, SP, 2002.

\begin{tabular}{|c|c|c|c|c|c|c|}
\hline \multirow{3}{*}{ Déficits } & \multirow{3}{*}{ Evolução } & \multicolumn{4}{|c|}{ Grupos } & \multirow{3}{*}{$\begin{array}{c}\text { Nível } \\
\text { descritive } \\
\text { (p-value) }\end{array}$} \\
\hline & & \multicolumn{4}{|c|}{ Controle Experimental } & \\
\hline & & $\mathbf{N}$ & $\%$ & $\mathbf{N}$ & $\%$ & \\
\hline \multirow[t]{3}{*}{ Déficit 1} & Progrediu & 6 & 42,86 & 11 & 78,57 & 0,107 \\
\hline & Não alterou & 6 & 42,86 & 3 & 21,43 & \\
\hline & Regrediu & 2 & 14,28 & - & - & \\
\hline \multirow[t]{2}{*}{ Déficit 2} & Progrediu & 4 & 28,57 & 9 & 64,29 & 0,058 \\
\hline & Não alterou & 10 & 71,43 & 5 & 35,71 & \\
\hline \multirow[t]{3}{*}{ Déficit 3} & Progrediu & 6 & 42,86 & 13 & 92,86 & 0,018 \\
\hline & Não alterou & 7 & 50,00 & 1 & 7,14 & \\
\hline & Regrediu & 1 & 7,14 & - & - & \\
\hline \multirow[t]{3}{*}{ Déficit 4} & Progrediu & 6 & 42,86 & 12 & 85,71 & 0,043 \\
\hline & Não alterou & 5 & 35,71 & 2 & 14,29 & \\
\hline & Regrediu & 3 & 21,43 & - & - & \\
\hline \multirow[t]{2}{*}{ Déficit 5} & Progrediu & 8 & 57,14 & 14 & 100,00 & 0,008 \\
\hline & Não alterou & 6 & 42,86 & - & - & \\
\hline \multirow[t]{3}{*}{ Déficit 6} & Progrediu & 11 & 78,57 & 14 & 100,00 & 0,186 \\
\hline & Não alterou & 2 & 14,29 & - & - & \\
\hline & Regrediu & 1 & 7,14 & - & - & \\
\hline \multirow[t]{2}{*}{ Déficit 7} & Progrediu & 13 & 92,86 & 14 & 100,00 & 0,500 \\
\hline & Não alterou & 1 & 7,14 & - & - & \\
\hline \multirow[t]{3}{*}{ Déficit 8} & Progrediu & 4 & 28,57 & 11 & 78,57 & 0,026 \\
\hline & Não alterou & 9 & 64,29 & 3 & 21,43 & \\
\hline & Regrediu & 1 & 7,14 & - & - & \\
\hline \multirow[t]{3}{*}{ Déficit 9} & Progrediu & 10 & 71,43 & 12 & 85,71 & 0,501 \\
\hline & Não alterou & 3 & 21,43 & 2 & 14,29 & \\
\hline & Regrediu & 1 & 7,14 & - & - & \\
\hline \multirow[t]{2}{*}{ Déficit 10} & Progrediu & 4 & 28,57 & 10 & 71,43 & 0,023 \\
\hline & Não alterou & 10 & 71,43 & 4 & 28,57 & \\
\hline \multirow[t]{2}{*}{ Déficit 11} & Progrediu & 3 & 30,00 & 5 & 50,00 & 0,361 \\
\hline & Não alterou & 7 & 70,00 & 5 & 50,00 & \\
\hline \multirow[t]{2}{*}{ Déficit 12} & Progrediu & 2 & 20,00 & 6 & 54,55 & 0,119 \\
\hline & Não alterou & 8 & 80,00 & 5 & 45,45 & \\
\hline \multirow[t]{2}{*}{ Déficit 13} & Progrediu & - & - & 3 & 50,00 & 0,357 \\
\hline & Não alterou & 2 & 100,00 & 3 & 50,00 & \\
\hline \multicolumn{2}{|l|}{ Déficit 14} & - & - & - & - & $* * *$ \\
\hline \multirow[t]{2}{*}{ Déficit 15} & Progrediu & 1 & 50,00 & 6 & 100,00 & 0,250 \\
\hline & Não alterou & 1 & 50,00 & - & - & \\
\hline \multirow[t]{2}{*}{ Déficit 16} & Progrediu & 1 & 7,14 & 10 & 71,43 & $\overline{0,001}$ \\
\hline & Não alterou & 13 & 92,86 & 4 & 28,57 & \\
\hline
\end{tabular}

Verificamos que foram observadas diferenças estatisticamente significantes entre os grupos nos déficits 2, 3, 4, 5, 8, 10 e 16. Nesses déficits, entre os pacientes do grupo experimental houve maior índice de progresso na assunção do autocuidado em relação ao grupo controle. Nos déficits 1, 3, 4, 6, 8 e 9 houve pacientes que regrediram na evolução nos métodos de ajuda. Todos esses pacientes pertenciam ao grupo controle.
Influência das variáveis nível neurológico da lesão e locus de controle à saúde no progresso dos pacientes paraplégicos na assunção do autocuidado

Verificamos a evolução dos pacientes com os três níveis neurológicos de lesão, T1 a T6, T7 a T11 e T12 ou abaixo, em cada déficit identificado neste estudo, utilizando o teste Quiquadrado ( $p$-value $<0,05)$ e não houve diferença estatisticamente significante para os déficits identificados neste estudo.

Verificamos também a evolução dos pacientes com locus de controle interno e externo em cada déficit de autocuidado identificado neste estudo, utilizando o teste Quiquadrado ( $p$-value< $<0,05)$ e não houve diferença estatisticamente significante para os déficits identificados neste estudo, com exceção apenas do déficit 16 que apresentou associação entre locus de controle interno e progresso na assunção do autocuidado.

\section{DISCUSSÃO}

A problemática da eliminação intestinal nos indivíduos paraplégicos foi verificada em alguns estudos, como por exemplo, um estudo que teve por objetivo determinar a prevalência de sintomas relacionados à constipação em lesados medulares e elaborou uma série de questões relacionadas a esses sintomas que foram respondidas por 161 pacientes que já tinham superado a fase aguda da lesão. Os resultados revelaram que 69\% dos sujeitos referiam o uso regular de laxantes, supositórios ou enemas, 41\% gastavam mais de uma hora no processo de evacuação, 50\% revelaram distensão abdominal e $27 \%$ dos indivíduos revelaram sintomas de disrreflexia autonômica no momento de evacuarem, 33\% dos indivíduos usavam estimulação digital para evacuar e $21 \%$ referiram a necessidade de extração manual de fezes, mesmo estabelecendo um programa de rotina para a evacuação. Foi sugerido que as dificuldades com evacuação possam ser profilaticamente trabalhadas ${ }^{(5)}$.

Um estudo exploratório-descritivo realizado no município de Ribeirão Preto (SP), com 27 pacientes que sofreram trauma raquimedular teve como um dos objetivos conhecer as complicações do funcionamento intestinal e as práticas de autocuidado, bem como relacionar o número de internações com informações sobre a reabilitação intestinal. As principais complicações intestinais relatadas pelos pacientes foram constipação/ impactação de fezes (50\%), e sangramento (20,6\%). A massagem abdominal foi a prática de autocuidado para o funcionamento intestinal mais utilizada $(48,1 \%)$. Apresentaram mais de uma internação 59,3\% dos pacientes, e apenas $22 \%$ receberam informações sobre alterações intestinais. As autoras destacam a importância de haver uma equipe interdisciplinar preparada para atuar com os pacientes com lesão medular durante a 
hospitalização( ${ }^{(6)}$.

Em outro trabalho, um programa de reabilitação ideal para lesados medulares que abrangesse os problemas de incontinência fecal e obstipação, evitando as complicações decorrentes, foi discutido. Acredita-se que o programa deva conter história prévia desses pacientes, exame físico, laboratoriais e radiológicos que possam definir limites e possibilidades. Deve-se ter como meta a continência de fezes, a evacuação independente e a prevenção de problemas gastrointestinais. Julga-se importante a implementação de um programa intestinal individualizado que deve conter uma dieta equilibrada com fibras, vegetais e frutas e adequada ingestão hídrica entre 2000 e 3000 $\mathrm{ml} /$ dia. O programa intestinal abrange a técnica de estimulação digital, a manobra de Valsalva e a massagem abdominal através do curso do cólon, salienta a importância do posicionamento sentado em assento confortável, evitando lesões de pele. Concluiu-se que os estudos desses problemas é uma área fértil de investigação e pesquisa e que inovações trarão melhora na qualidade de vida desses pacientes ${ }^{(7)}$.

Neste estudo buscamos a elaboração de um programa intestinal adequado, mas acima de tudo, verificar se o programa de ensino conseguiria levar os pacientes paraplégicos à assunção do autocuidado. Acreditamos que conseguimos atingir esse objetivo.

\section{CONSIDERAÇÕES FINAIS}

Em relação ao tamanho da amostra, foram realizados vários testes estatísticos a fim de se verificar possíveis tendenciosidades nos grupos, sendo que os testes não demonstram vieses; os pacientes foram originários de duas instituições, sendo que as condições do serviço de ambas é muito semelhante, a equivalência das mesmas foi, todavia, inferida; houve perda de oito pacientes, o que em estudo experimental pode influenciar nos resultados.

\section{CONCLUSÕES}

Em relação ao nível neurológico da lesão, a maioria dos pacientes apresentou lesão torácica alta; em relação ao lócus de controle à saúde, observamos que em 13 pacientes da amostra o locus de controle é interno e, igualmente, em 13 pacientes o locus de controle é externo. Há homogeneidade entre os grupos controle e experimental da amostra estudada.

A aplicação do programa educativo para o grupo experimental mostrou que na avaliação final, mais pacientes deste grupo encontravam-se nas categorias de menor dependência da enfermagem, segundo os métodos de ajuda necessários. Os paraplégicos do grupo experimental progrediram na assunção do autocuidado em todos os déficits identificados neste estudo.

Segundo as categorias progrediu, não alterou e regrediu houve maior índice de progresso na assunção do autocuidado do grupo experimental em relação aos pacientes do grupo controle nos déficits 2, 3, 4, 5, 8, 10 e 16. Alguns pacientes regrediram na evolução nos métodos de ajuda e todos pertenciam ao grupo controle.

Pelo teste de Quiquadrado foi demonstrado que não houve influência do nível neurológico da lesão e também do lócus de controle à saúde na assunção do autocuidado.

Os resultados permitem-nos aceitar a hipótese deste trabalho: Paraplégicos submetidos a um programa educativo visando o autocuidado relacionado aos déficits identificados na eliminação intestinal, conseguem progredir na assunção do autocuidado.

\section{REFERÊNCIAS}

1. Smeltzer SC, Bare BG. Brunner \& Suddarth: tratado de enfermagem médico-cirúrgica. 9a ed. Rio de Janeiro: Guanabara Koogan; 2002. p.1613-26.

2. Lianza S, Casalis MEP, Greve JMD, Eichberg, R. A lesão medular. In: Lianza S, oordenador. Medicina de reabilitação. 2a ed. Rio de Janeiro: Guanabara Koogan; c1995. p. 304-26.

3. Tonello AS. Aspectos de reeducação intestinal em lesados medulares [tese]. São Paulo: Universidade Federal de São Paulo. Escola Paulista de Medicina; 1999.

4. Orem DE, Taylor SG, Renpenning KM . Nursing: concepts of practice. St. Louis: Mosby; 1995.

5. Harari D, Sarkarati M, Gurwitz JH, McGlinchey-Berroth G, Minaker KL. Constipation-related symptoms and bowel program concerning individuals with spinal cord injury. Spinal Cord. 1997; 35 (6): 394-401.

6. Furlan MLS, Caliri MHL. Complicações do funcionamento intestinal e práticas de auto-cuidado em pacientes com trauma raquimedular. Coluna/Columna. 2005; 4 (1): 16-21.

7. Stiens SA, Bergman SB, Goetz LL. Neurogenic bowel dysfunction after spinal cord injury: clinical evaluation and rehabilitative management. Arch Phys Med Rehabil. 1997; 78 (3 Suppl): S86-102. 